\title{
The Commutation Property of a Stationary, Axisymmetric System
}

\author{
BRANDON CARTER \\ Institute of Theoretical Astronomy, Cambridge, England
}

Received December 12, 1969

\begin{abstract}
It is shown that in studies of space-time systems which are both stationary and axisymmetric, no generality is lost by considering only cases where the stationary and axisymmetric actions (or equivalently the two corresponding Killing vector fields) commute.
\end{abstract}

\section{Introduction}

In discussions of the properties of stationary axisymmetric systems (e.g. in the derivation of Papapetrou's Theorem $[1,2]$ ) it is normally assumed at the outset that the stationary and axisymmetric actions (or equivalently the two corresponding Killing vector fields) commute. Since it is perhaps not immediately obvious (particularly when curved space-time is involved) that this is always justifiable, and since stationary axisymmetric models are so widely used (not only in relativistic and non-relativistic astrophysics, but also in many other branches of physics) it seems worthwhile to demonstrate formally that such an assumption never involves any loss of generality. This is the purport of the theorem which is stated and proved here (which applies whenever the space is sufficiently well behaved to have a continuous Riemann tensor).

The relevant basic properties of axisymmetry and stationary symmetry actions are set out in the definitions and propositions of Sections 3 and 4 in terms of the mathematical framework in Section 2. The theorem itself is given in Section 5.

\section{Actions on $\mathscr{M}$}

Throughout the whole of this work, it is to be understood that $\mathscr{M}$ (the subject of discussion) is a connected, $C^{3} n$-dimensional manifold on which is defined a $C^{2}$ Riemannian or pseudo-Riemannian metric and on which further structure (e.g. a Maxwell field, or a field representing stellar matter) may also have been specified. (In most of the physical applications one has in mind, $n$ will be 3 or 4 .) 
We shall start by recapitulating some standard notation.

The statement that $\mathscr{M}$ is a spacetime will mean that its metric has hyperbolic-normal signature, i.e., more explicitly, that it is non-degenerate with signature $(n-2)$. When this condition holds a submanifold or tangent element will be said to be spacelike if its induced metric is positive definite, null if its induced metric is degenerate, and timelike otherwise.

If $G$ is a Lie group, then a $C^{3}$ mapping $\pi: G \times \mathscr{M} \rightarrow \mathscr{M}$ will be referred to as an action of $G$ on $\mathscr{M}$ if the restricted mappings $\pi_{g}: \mathscr{M} \rightarrow \mathscr{M}$ defined for each $g \in G$ by $\pi_{g} m=\pi(g, m), m \in \mathscr{M}$ satisfy the following axioms:

(1) $\pi_{e}$ is the identity mapping when $e$ is the unit element of $G$,

(2) for any $g, g^{\prime} \in G, \pi_{g} \pi_{g}^{\prime}=\pi_{g g^{\prime}}$.

The action $\pi$ will be said to be effective if the converse of axiom (1) is also true, i.e. if $\pi_{g}$ is the identity mapping only when $g=e$. For some given $m_{0} \in \mathscr{M}$ the set of points $\mathscr{I}\left(\pi, m_{o}\right) \equiv\left\{m \in \mathscr{M}:\left(\exists g \in G: m=\pi_{g} m_{0}\right)\right\}$ will be referred to as the surface of transitivity of $\pi$ through $m_{0}$; if $\mathscr{I}\left(n, m_{0}\right)=m_{0}$ then $m_{0}$ will be said to be a fixed point of $\pi$.

$\mathscr{M}$ will be said to be invariant under an action $\pi$ of $G$ if for each $g \in G$ the mapping $\pi_{g}$ is an isometry which also preserves whatever further structure (e.g. a Maxwell field or a stellar matter field) has been specified on $\mathscr{M}$. If $\mathscr{M}$ is invariant under an effective action $G$ then $G$ will be said to be an invariance group of $\mathscr{M}$.

Let $\pi^{G}: G \times \mathscr{M} \rightarrow \mathscr{M}$ and $\pi^{H}: H \times \mathscr{M} \rightarrow \mathscr{M}$ be actions of $G, H$ respectively on $\mathscr{M}$. $\pi^{H}$ will be said to be a subaction of $\pi^{G}$ if $H$ is a subgroup of $G$ and $\pi_{h}^{H}=\pi_{h}^{G}$ for all $h \in H \subset G . \pi^{H}$ will be said to commute with $\pi^{G}$ if $\pi_{g}^{G} \pi_{h}^{H}=\pi_{h}^{H} \pi_{g}^{G}$ for all $g \in G, h \in H$. It can easily be shown that if (and only if ) $\pi^{G}$ and $\pi^{H}$ commute the law $\pi^{G} \oplus \pi^{H}(g, h, m)=\pi_{g}^{G} \pi_{h}^{H} m$ defines an action $\pi^{G} \oplus \pi^{H}: G \times H \times \mathscr{M} \rightarrow \mathscr{M}$ of the direct product group $G \times H$ on $\mathscr{M}$. When it is well defined, i.e. when $\pi^{G}$ and $\pi^{H}$ commute, this action $\pi^{G} \oplus \pi^{H}$ will be described as the direct product of the subactions $\pi^{G}$ and $\pi^{H}$.

\section{Axisymmetric Systems}

For general mathematical purposes it is necessary to distinguish axisymmetric spaces within the more general class of cyclicly symmetric spaces, although as the comments at the end of this section make clear, the distinction does not arise in ordinary astrophysical contexts. These terms are defined as follows:

Definition 1. $\mathscr{M}$ will be said to be cyclicly symmetric (under the action $\pi^{C}$ ) if it is invariant under an effective action $\pi^{C}: S O(2) \times \mathscr{M} \rightarrow \mathscr{M}$ of the one parameter cyclic group $S O(2)$. When these conditions are satisfied $\pi^{C}$ will be said to be a cyclic symmetry action. 
Definition 2. $\mathscr{M}$ will be said to be axisymmetric (under the action $\pi^{A}$ with axis $\mathscr{N}^{A}$ ) if it is cyclicly symmetric under an effective action $\pi^{A}$ such that there is an imbedded $(n-2)$-surface $\mathscr{N}^{A}$, called the axis of $\pi^{A}$, which is the fixed point set of $\pi^{A}$. When these conditions are satisfied $\pi^{A}$ will be said to be an axial symmetry action.

By considering the family of 2-surfaces swept out by the normals to $\mathscr{N}^{A}$ under the maps $\pi_{\varphi}^{A}$ as $\varphi \in S O(2)$ varies (and, for Prop. 1, using the fact that, since the Riemann tensor is continuous, a Killing vector field which vanishes on an open subset must vanish everywhere in $\mathscr{M}$ ) one can readily prove:

Proposition 1. There is at most one action $\pi^{A}$ under which $\mathscr{M}$ is axisymmetric with a given axis $\mathscr{N}^{A}$.

Proposition 2. If $\mathscr{M}$ is a spacetime, and is axisymmetric under an action $\pi^{A}$ with axis $\mathscr{N}^{A}$, then $\mathscr{N}^{A}$ is timelike.

With the aid of the standard fixed point theorem for a 2-sphere one can straightforwardly establish the following results:

Proposition 3. Let $\mathscr{M}$ be either (a) a positive definite metric space with $n \leqq 3$ or (b) a space-time with $n \leqq 4$. Then if $\mathscr{M}$ is cyclicly symmetric under an action $\pi^{A}$ with fixed points, it follows that $\mathscr{M}$ is axisymmetric under $\pi^{A}$.

Proposition 4. Let $\mathscr{M}$ be either (a) a positive definite metric space with $n \leqq 3$ which is asymptotically Euclidean or (b) a spacetime with $n \leqq 4$ which is asymptotically Minkowskian in.spacelike directions. Then if $\mathscr{M}$ is cyclicly symmetric under an action $\pi^{A}$, it follows that $\pi^{A}$ has fixed points, and hence (by Prop. 3) that $\mathscr{M}$ is axisymmetric under $\pi^{A}$.

Owing to the absence of appropriate fixed point theorems for higher dimensional spheres, it is not possible to generalise either of these two propositions to higher values of $n$ (e.g. it is easy to construct counterexamples to the analogous statements for positive definite metric spaces with $n=4$ by using the Hopf fibration of the 3 -sphere).

Nevertheless the cases which are covered are sufficient for direct astrophysical applications. Thus Prop. 4 shows why, in ordinary astrophysical problems, cases which are cyclicly symmetric without being axisymmetric never arise.

\section{Stationary Systems}

The standard definition of the term stationary is as follows:

Definition 3. $\mathscr{M}$ will be said to be stationary (under an action $\pi^{S}$ ) if it is a spacetime and is invariant under an action $\pi^{S}: R(1) \times \mathscr{M} \rightarrow \mathscr{M}$, 
of the one parameter linear translation group $R(1)$, such that the trajectories are timelike curves. When these conditions are satisfied $\pi^{S}$ will be said to be a stationary symmetry action.

This definition does not require that a stationary symmetry action be effective. However, the stationary symmetry actions which occur in ordinary physical and astrophysical problems always are effective since stationary symmetry actions which are not effective (i.e. the cyclicly stationary cases, which are exemplified by N.U.T. space [3] without the Taub continuation) automatically involve large scale causality violation (i.e. the existence of closed timelike lines.)

A stationary symmetry action $\pi^{S}$ gives rise to a one parameter family of isometries $\pi_{t}^{s}: \mathscr{M} \rightarrow \mathscr{M}, t \in R(1)$, and the corresponding cross section $d /\left.d t\left(\pi_{t}^{s} m\right)\right|_{t=0}, m \in \mathscr{M}$ of the tangent bundle $T(\mathscr{M})$ is a timelike Killing vector field which we shall denote, for brevity, by $\dot{\pi}^{S}$. Conversely any timelike Killing vector field on $\mathscr{M}$ generates a unique stationary symmetry action on $\mathscr{M}$. This well known result may be stated briefly as:

Proposition 5. There is a canonical one-one correspondence $\left(\pi^{S} \leftrightarrow \dot{\pi}^{S}\right)$ between stationary symmetry actions and timelike Killing vector fields on $\mathscr{M}$.

\section{Stationary-Axisymmetric Systems}

The main result of this work is contained in the following statement:

Theorem. Let $\mathscr{M}$ be both stationary and axisymmetric. Then $\mathscr{M}$ is invariant under an action of the form $\pi^{S} \oplus \pi^{A}: R(1) \times S O(2) \times \mathscr{M} \rightarrow \mathscr{M}$ of the 2-parameter Abelian cylindrical group $R(1) \times S O(2)$ where $\pi^{S}$ is a stationary symmetry action and $\pi^{A}$ an axial symmetry action which commutes with $\pi^{s}$.

This result can be derived immediately from the rather more elaborate proposition which follows. This proposition and its proof are given in detail because the situation is slightly less straightforward than the theorem as stated above might suggest, due to the fact that it is not necessarily true that a given axial symmetry action commutes with a given stationary symmetry action.

Proposition 6. Let $\mathscr{M}$ be stationary under an action $\pi^{B}$ and axisymmetric under an action $\pi^{A}$ with axis $\mathscr{N}^{A}$. Then $\mathscr{M}$ is stationary under an action $\pi^{S}$ which commutes with $\pi^{A}$, and either $\pi^{S}$ can be chosen to coincide with $\pi^{B}$ or else $\mathscr{M}$ is invariant under an effective action $\pi: G \times \mathscr{M} \rightarrow \mathscr{M}$, (containing $\pi^{A}, \pi^{C}, \pi^{S}$ as subactions), of a 4 or higher dimensional group $G$, with 3 or higher dimensional surfaces of transitivity almost everywhere. 
Proof. Let us describe the elements $\varphi \in S O(2)$ by a circular coordinate running from 0 to $2 \pi$ in the usual way, so that $\varphi=0$ is the unit element and $\varphi=\pi$ is the element corresponding to half a complete rotation.

The trajectories of $\pi^{A}$ must be imbedded circles except on $\mathscr{N}^{A}$ and since $\mathscr{M}$ is connected and $\pi^{A}$ is effective it follows that for any $m \in \mathscr{M}, m \notin \mathscr{N}^{A}$ the point $\pi_{\varphi}^{A} m$ sweeps out the corresponding circle once and once only as $\varphi$ runs from 0 to $2 \pi$. Now consider the effect of the map $\pi_{\varphi}^{A}: T(\mathscr{M}) \rightarrow T(\mathscr{M})$ (induced on the tangent space $T(\mathscr{M})$ by $\pi_{\varphi}^{A}: \mathscr{M} \rightarrow \mathscr{M}$ ) on a vector $\xi \in T\left(m_{0}\right)$ where $m_{0} \in \mathscr{N}^{A}$. Such a vector $\xi$ can be uniquely expressed in the form $\xi=\xi^{\prime \prime}+\xi^{\perp}$ where $\xi^{\prime \prime}$ is parallel and $\xi^{\perp}$ orthogonal to $\mathscr{N}^{A}$. Clearly $\pi_{\varphi}^{A} \xi^{\prime \prime}=\xi^{\prime \prime}$ for all $\varphi \in S O(2)$ whereas it follows from the preceeding remarks, that $\pi_{\varphi}^{A} \xi^{\perp}$ must sweep out a circle (in the 2-plane of $T\left(m_{0}\right)$ orthogonal to $\mathscr{N}^{A}$ ) once and once only as $\varphi$ varies from 0 to $2 \pi$, and hence that $\pi_{\pi}^{A} \xi^{\perp}=-\xi^{\perp}$. Hence $\xi+\pi_{\pi}^{A} \xi$ is tangent to $\mathscr{N}^{A}$.

Now since $\mathscr{M}$ is invariant under $\pi^{A}$ and since $\dot{\pi}^{C}$ is a timelike Killing vector field, it follows that $\pi_{\varphi}^{A} \dot{\pi}^{B}$ is a timelike Killing vector field for all $\varphi \in S O(2)$ and moreover it must have the same time orientation as $\dot{\pi}^{B}$ since $S O(2)$ is connected.

Let us temporarily make the supposition that $\dot{\pi}^{B}$ is not everywhere tangent to $\mathscr{N}^{A}$, or more specifically that there exists a point $m_{0} \in \mathscr{N}^{A}$ such that $\dot{\pi}^{B}\left(m_{0}\right)^{\perp}$ is non zero. Then $\pi_{\pi}^{A} \dot{\pi}^{B}\left(m_{0}\right), \pi_{\pi / 2}^{A} \dot{\pi}^{B}\left(m_{0}\right)$ and $\dot{\pi}^{B}\left(m_{0}\right)$ are three non vanishing and independent vectors and hence $\pi_{\pi}^{A} \dot{\pi}^{B}$, $\pi_{\pi / 2}^{A} \dot{\pi}^{B}$, and $\dot{\pi}^{B}$ are three independent Killing vector fields which are independent not only of each other but also of the Killing vector field $d /\left.d \varphi \pi_{\varphi}^{A}(m)\right|_{\varphi=0}, m \in \mathscr{M}$, which generates $\pi^{A}$, since this latter field vanishes at $m_{0}$. It follows that these four Killing vector fields together must generate an action $\pi$ of a group $G$ of 4 or higher dimensions and that the surface of transitivity of $\pi$ through $m_{0}$ is of three or higher dimensions. Hence from general properties of group actions, the surfaces of transitivity must be of three or higher dimensions almost everywhere on $\mathscr{M}$.

Now (independently of the above supposition) consider the vector field, $\dot{\pi}^{S}$ say, defined as $\dot{\pi}^{S}=1 / 2\left(\dot{\pi}^{B}+\pi_{\pi}^{A} \dot{\pi}^{B}\right)$. Since $\dot{\pi}^{B}$ and $\pi_{\pi}^{A} \dot{\pi}^{B}$ are timelike Killing vector fields and since they have the same time orientation, it follows that $\dot{\pi}^{S}$ is also a timelike Killing vector field, and hence generates a canonically determined stationary symmetry action $\pi^{S}$ say (by Prop. 5).

Any mapping $\pi_{t}^{S}, t \in R(1)$ transforms the action $\pi^{A}$ into an action $\pi_{t}^{S}\left(\pi^{A}\right): S O(2) \times \mathscr{M} \rightarrow \mathscr{M}$ according to the rule $\pi_{t}^{S}\left(\pi^{A}\right)(\varphi, m)=\pi_{t}^{S} \pi_{\varphi}^{A} \pi_{-t}^{S} m$, $\varphi \in S O(2), m \in \mathscr{M}$ and since $\mathscr{M}$ is invariant under $\pi_{t}^{S}$ and since $\pi^{A}$ is an axisymmetry action, it follows that $\pi_{t}^{S}\left(\pi^{A}\right)$ is also an axisymmetry action. Moreover $\pi_{t}^{S}$ leaves $\mathscr{N}^{\boldsymbol{A}}$ invariant since it follows from our earlier working that $\dot{\pi}^{S}$ is tangent to $\mathscr{N}^{A}$, and hence the axisymmetry action $\pi_{t}^{S}\left(\pi^{A}\right)$ has 
the same axis $\mathscr{N}^{A}$ as the original axisymmetry action $\pi^{A}$. It follows (by Prop. 1) that $\pi_{t}^{S}\left(\pi^{A}\right)=\pi^{A}$, which implies (since this is true for all $t$ ) that $\pi^{S}$ commutes with $\pi^{A}$.

At this point we have already gone far enough to establish the main theorem. To complete the proof of Prop. 6 consider the case where the supposition required for proving the existence of $G$ does not hold, i.e. the case where $\dot{\pi}^{B}$ is everywhere tangent to $\mathscr{N}^{A}$. Then $\dot{\pi}^{B}$ has all the properties of $\dot{\pi}^{S}$ which were used in proving that $\pi^{S}$ commutes with $\pi^{A}$, and hence $\pi^{B}$ must also commute with $\pi^{A}$ (with the further implication that $\pi_{\pi}^{A} \dot{\pi}^{B}=\dot{\pi}^{B}$, so that $\pi^{B}$ is in fact identical with $\pi^{S}$ ). This completes the proof.

Acknowledgement. I should like to thank Kip Thorne for directing my attention to the need for a formal clarification of this matter.

\section{References}

1. Papapetrou, A.: Ann. Inst. Poincaré 4, 83 (1966).

2. Carter, B.: J. Math. Phys. 10, 70 (1969).

3. Newman, E., Tamborino, L., Unti, T.: J. Math. Phys. 4, 915 (1963).

B. Carter

Institute of Theoretical Astronomy

Cambridge, England 